$2019-10$

\title{
Application of Quality of Experience in Networked Services: Review, Trend \& Perspectives
}

\section{Laghari, A}

http://hdl.handle.net/10026.1/12432

\subsection{7/s11213-018-9471-x}

Systemic Practice and Action Research

Springer Verlag

All content in PEARL is protected by copyright law. Author manuscripts are made available in accordance with publisher policies. Please cite only the published version using the details provided on the item record or document. In the absence of an open licence (e.g. Creative Commons), permissions for further reuse of content should be sought from the publisher or author. 


\title{
Application of Quality of Experience in Networked Services: Review, Trend \& Perspectives
}

\author{
Asif Ali Laghari ${ }^{1 *}$, Hui $\mathrm{He}^{1^{*}}$, Muhammad Shafiq ${ }^{1}$ and Asiya $\mathrm{Khan}^{2}$ \\ ${ }^{1}$ School of Computer Science \& Technology, Harbin Institute of Technology \\ ${ }^{2}$ University of Plymouth, United Kingdom \\ Email: asiflaghari, hehui, muhammadshafiq [@hit.edu.cn], asiya.khan@plymouth.ac.uk \\ *Correspondence: asiflaghari@hit.edu.cn, hehui@hit.edu.cn; Tel.: +8615636147179
}

\begin{abstract}
Over the last decade, there has been an exponential growth of services over the Internet. Services such as online gaming are gaining popularity, whereas, Voice over Internet (VoIP) has been well established. Storage, management and processing of data have moved away from the devices to the cloud. Quality of experience (QoE) defined as the user's perception of service quality is utilized in different technologies, services and products for improving end user satisfaction and provides quality of service (QoS) according to their demands. In this paper, we describe the utilization of QoE in different technologies, services and products for improving end user satisfaction. Specifically, we present the latest developments in QoE in multimedia services, network management, VoIP, web development, games and cloud computing. Finally, we discuss the current research, solutions and present open issues of future research in QoE.
\end{abstract}

Keywords: Network Services; Cloud Computing; Quality of Experience (QoE); QoE frameworks;

\section{Introduction}

With the advancement of technology in the modern era and global competition in businesses that generate more revenues from markets, vendor's change their strategy to attract customers and provide better service to become a permanent user of their services. Many organizations use Quality of Experience (QoE) to find customer needs and try to provide services according to their needs. QoE is users' evaluation of data about network and services provided by the network (Dong et al. 2014). The definition of QoE is "the degree of delight or annoyance of the user of an application or service" (Callet et al. 2013). Further, QoE defined in a general perspective as "a measurement of customer satisfaction or customer performance depending on objective or subjective measure of using any service or product" (Laghari et al. 2015). There are different definitions of QoE provided by academia and industry. The International Telecommunication Union (ITU-T) defines QoE (ITU 2007) as "the overall acceptability of an application or service, as perceived subjectively by the end-use". Laghari and Connnelly (2012) define QoE as "a blueprint of all human subjective and objective quality needs and experiences arising from the interaction of a person with technology and with business entities in a particular context". Vendors of products use QoE to get information about user needs and demand, which are changed over time (Nourikhah et al. 2016). They use interviews, web based surveys and questionnaires to get subjective information from users about products or services (Laghari et al. 2018). Organizations focus on the user satisfaction, efficiency, effectiveness, learning and enjoyment about products or services (Soldani and 
Cuny 2007). There are two type of QoE used to observe user perception, one is subjective and the second is objective (Khan et al. 2010). Web surveys, interviews, questionnaires and complaints are used to collect subjective QoE. Objective QoE can be captured by using two methods (i) technical quality of service (QoS) data and (ii) cognitive systems and human physiological tests (Laghari et al. 2018). Sometimes users provide negative feedback due to their greedy nature to get more quality services mentioned in the service level agreement (SLA) and also due to less technical knowledge so it is a big problem for vendors to distinguish positive and negative QoE. Vendors do not want negative feedback from the user because they spend a lot of money on product development and service delivery to customers so they desire positive responses to improve more quality of product or service according to their needs (Varela et al. 2014). ITU provides standards known as Mean Opinion Score (MOS) to collect subjective responses from users shown in Table 1 (ITU 2002).

Table 1. Mean opinion score (ITU 2002)

\begin{tabular}{|c|c|c|}
\hline MOS & Quality & Perception \\
\hline 5 & Excellent & Imperceptible \\
\hline 4 & Good & Perceptible \\
\hline 3 & Fair & Slightly annoying \\
\hline 2 & Poor & Annoying \\
\hline 1 & Bad & Very annoying \\
\hline
\end{tabular}

QoE is used in several fields such as in cloud computing for analysis of services, image quality testing, user perception of video streaming and online gaming. Telecom sector uses QoE to improve network services and multimedia services. The details of QoE utilization in different areas are presented in the next sections.

The paper will address the topic of QoE, state of the art of QoE and how QoE is utilized for multimedia services, for web development, for games, and in cloud computing. It surveys both subjective and objective quality assessment methodologies for the multimedia and network services, web development, cloud gaming and Voice over Internet Protocol (VoIP) together with their potential applications, such as system tuning and diagnosis. Limitation of previous work and challenges of future work also discussed. Further, we identify and discuss a set of open issues yet to be addressed, for accurate quality estimation of QoE of multimedia services, web development, cloud gaming, network services and cloud computing.

This paper is based on the QoE survey and review in different areas and is divided into 8 sections. Section two is based on QoE frameworks for Multimedia services, section three provides details of the role of QoE in the improvement of Network services. Similarly, sections four, five, six \& seven are based on the QoE utilization in VoIP, Web development, Games and Cloud computing respectively. Finally, in section eight, we present the open research questions in this field.

\section{Quality of Experience for Multimedia Services}

Nowadays there is a rapid growth in multimedia services like video on demand (VOD), video teleconference (VTC), online tutorials and Internet TV (Chen et al. 2015, Laghari et al. 2018, 2017). 
Different devices, like smart phones, IPAD/tablets and laptops utilize heterogeneous protocols and multimedia services, face QoS problems (Zhao et al 2017, Ljubojević et al. 2013). Researcher's developed a QoE framework for the assessment of the user's satisfaction for video quality and delivery of services improving QoS for multimedia services. Venkataraman and Chatterjee (2011) propose a multimedia framework called "Mintmos: A lightweight, real time, no reference framework for Multimedia services. The Mintmos framework is based on the subjective quality of experience of four parameters - encoding video bit rate, the severity of impact, motion complexity and loss fraction. The Mintmos framework provides quantitative analysis of network level QoS (NQoS) and application level (AQoS) to calculate QoE scores. A similar framework for video services based on AQoS only is presented in (Kawano et al. 2010).

Niche vendors also delivered a way out for measurement of QoE for multimedia services. Niche vendors mostly focus on objective QoE (i.e. QoS) instead of subjective QoE. Key parameters for assessment of QoE using perceptual evaluation of speech quality (PESQ) technique use peak signal to noise ratio (PSNR) [Laghari et al. 2017].

A QoM Framework which provides QoE assessment for multimedia services has been proposed by Laghari et al (2012). Its main focus is on the subjective evaluation of QoE based on QoS parameters reporting tool alerts to the network administrator in the event of degradation in QoE. However, it does not support automatic policy change based on dynamic user requirements over the time.

Our previous work proposed EQoM, which is an advancement of QoE monitoring tool, uses agent technology to capture objective QoE/QoS and subjective QoE (Laghari et al. 2013). Agent technology is defined as "An autonomous agent is a system situated within a part of an environment, which senses the environment and acts on it, in pursuit of its own agenda and so as to affect what it sees in the future". Agents run in the dynamic environment from the server to client to find available resources, measure network conditions, and report them to a central point and use resources for the specific task. Agents run from the video server to end user's device and capture QoS data of network traffic, resources utilization and configuration of user's device. This framework estimated both NQoS and AQoS and also provided results to end user about network degradation. The user has an option to submit subjective QoE at any time, which will be stored in the user profile for future use. After submission of user's feedback, the EQoM framework analyzes both the subjective and objective QoE, independent of whether or not the user submitted incorrect feedback or correct feedback when using services. If a user did not receive services according to the SLA then EQoM upgrades the user's policies to provide QoS according to SLA (Laghari et al. 2017). EQoM framework supports functionality such as QoE monitoring, evaluation, reporting, runtime policy change mechanism and service quality assurance as per user profile (policy) (Laghari et al. 2013, 2017).

A solution provider organization named as PeerApp has developed a QoE content delivery acceleration tool (http://www.peerapp.com/quality-of-experience/ 2016). This tool manages Internet contents such as live video streaming, accelerates the network traffic for subscribers and helps to eliminate stalls and buffering during video playback. This contributes to improved customer QoE, which is a customer satisfaction driver. The comparison of results using the PeerApp tool with normal user and installation method is given in Figures 1 and 2 respectively. 


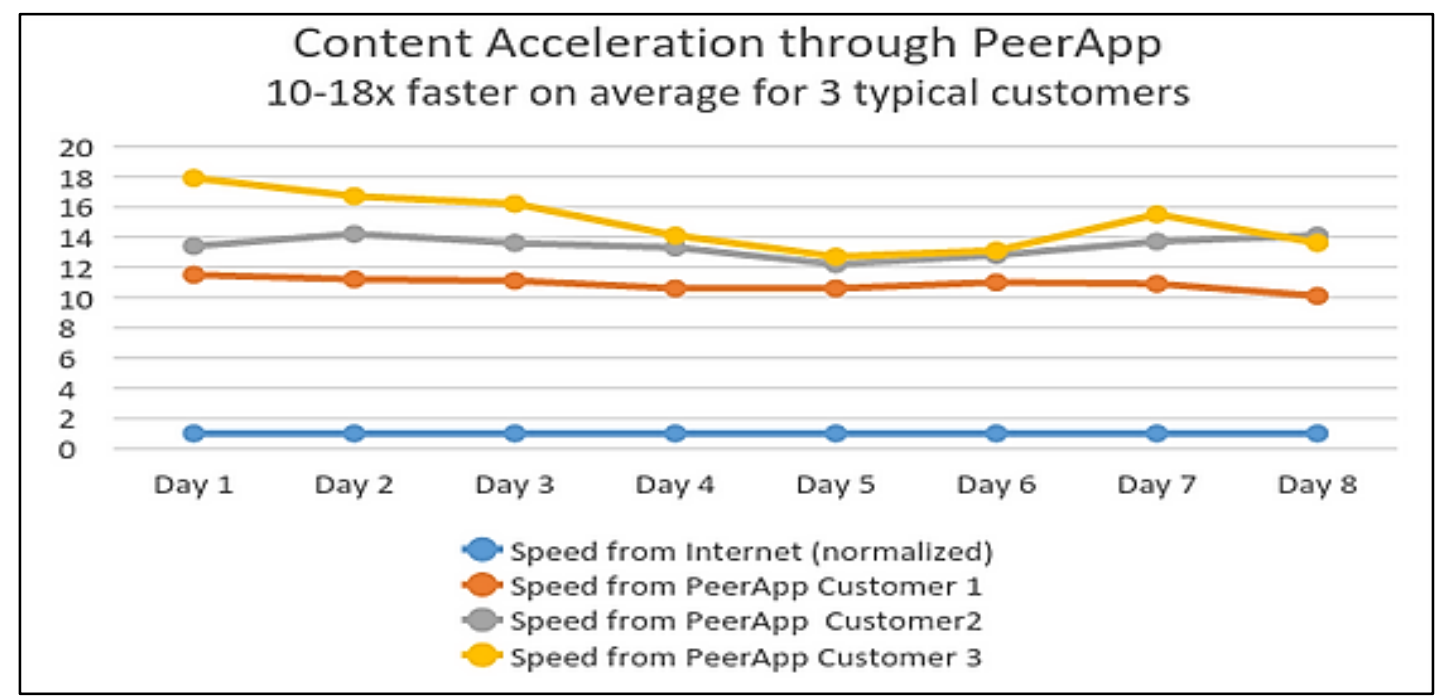

Figure 1. Comparison of network traffic with PeerApp to normal user (http://www.peerapp.com/qualityof-experience/ 2016)

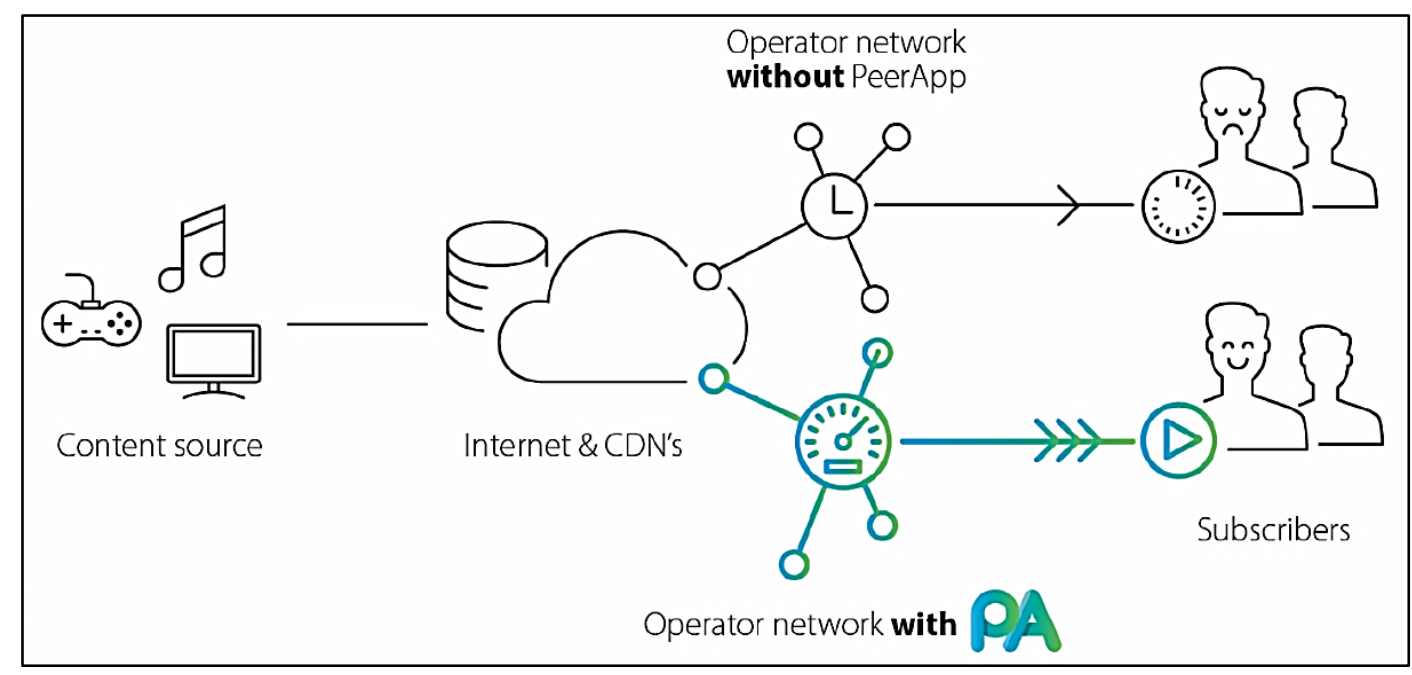

Figure 2. Deployment QoE tool of PeerApp tool (http://www.peerapp.com/quality-of-experience/ 2016)

\subsection{Challenges and limitations}

The work presented in Oche et al. (2017) concludes that the bit rate, data rate, frame rate, throughput, packet loss and delay are the parameters that impact multimedia service QoE (Laghari et al. 2018). Analysis of the previous QoE frameworks for multimedia services show that some frameworks are limited in how they capture accurate QoE, report to administrator and support one type of QoE monitoring (Varga et al. 2017). Only a few of them support both subjective and objective QoE analysis but are unable to change network policy of users on their feedback. The limited functionality of previous 
work motivates to research into more accurate frameworks to capture both types of QoE, monitoring from the server to client and support network policy change according to user's need.

In the future, during the development of QoE frameworks researchers will face challenges such as monitoring of client device, the design of algorithms for analysis of accurate QoE and network policy change. Monitoring of client device is a concern for user's privacy and also developers to break the firewall of the client device. The greedy behavior of users force to give wrong feedback about the services which can be a challenge for developers to sort out with positive feedback and how network policy supports QoE.

\section{Quality of Experience for Network Services}

Several mobile companies use QoE for getting feedback from users to improve services according to the user's needs (Shaikh et al 2010). User mobility, limited bandwidth and signal quality is a big issue in cellular networks. In addition, users accessing videos on the small screen of mobile phones have limited resources in the devices (Laghari et al. 2011, Reichl 2010). Videos that are accessed by using the mobile phone via wireless networks have different codecs, bitrates and frame rates, so user do not receive QoS and join other networks (Khan et al. 2009, Baraković et al. 2013). Telecom sector uses QoE as a solution of this problem and hence, avoid violation of SLA by getting user feedback on their services (Varela et al. 2015, Menkovsk and Liotta 2012).

Sprint communication has launched new Epitiro 4150 probe to measure QoE for Ethernet, Wi-Fi and LTE. This can help enterprises and service providers to measure QoE with the single probe for fixed and mobile user, providing a broad perceptibility of the service experience. This is capable to measure parameters which affect the QoE, the performance of commercial services and popular applications such as Gmail, Facebook and YouTube. The network parameters include latency and speed in LTE, Wi-Fi and Ethernet (Spirent 2016).

Quality of experience framework for network services (QON) is a proposed framework for network services such as HTTP, FTP and RTP etc. This framework automatically monitors network traffic using agent technology. The QoE administration of framework contains QoE database, which contains previously collected QoE and SLA of a particular user with a profile. Automatic QoE collection method reduces the burden from user's to submit QoE and user are unaware of monitoring process of QON framework. The user has an option to submit his complaint on QoS degradation. Policy management function analyzes the previous policy, make changes if required and enforce updated policy for the user and QON framework automatically monitor services according to the newly enforced policy. This framework supports organizations to provide services according to SLA to users and evolve their needs in the future (Laghari et al. 2012).

User centric network management approach is used in network systems to provide reliable services according to SLA. The QoE is monitored to measure the quality of service and if the user did not get services according to SLA, user will receive compensation from the service provider. A conceptual framework for measuring and managing QoE in the network has been given by Alex et al for providing compensation to end user and his expectation from network (Mongi 2015). This framework focuses on the user needs and integrates the current networked system by providing facilities to customers. The QoE is also used for HTTP Adaptive Streaming (HAS) to provide quality of service to the premium user in 
network systems (Phan-Xuan and Kamioka (2016). HAS is implemented by the popular service provider such as You Tube, Netflix, etc. instead of RTP because HAS provides video traffic without packet loss. HAS adapts the bitrate of the video resulting in low QoE for premium users who are willing to pay for stable QoE. New machine learning techniques have been proposed for proper network management to predict and analyze usage of behavior and requirement of different type of users. This scheme, based on the machine learning technique will attempt to solve the problem of network utilization competition of users and distribute network bandwidth in a fair way between the premium and free users (Phan-Xuan and Kamioka (2016).

\subsection{Challenges and Limitations}

The study of QoE for network services show that the delivery of network services according to user needs and network management is still a problem for services providers. Heterogeneous devices with different configurations and network technologies change over time in a network from the users and service provider's side. New technologies are introduced such as LTE, 5G, etc., therefore, new QoE models are required for network management (Hossain and Hasan 2015). Due to increasing demand of network resources, it is cumbersome for network operators to manage resources on the basis of QoE. Network operators require increasing resources such as more bandwidth, higher link quality and less delay to satisfy user's high expectations of seamless communication.

\section{Quality of Experience for Voice over Internet Protocol (VoIP)}

VoIP offers another multimedia streaming service over the data networks (Lloret et al. 2012). VoIP calls are dialed on the data networks (Internet) by avoiding local telephone call system (Nihei et al. 2016, Jones et al. 2000). Over the Internet mostly users face QoS issues because of network traffic congestion, application software, audio codec and device (Papadopouli et al. 2016). QoE is used in VoIP service to measure the user satisfaction level during the call monitoring technical parameters such as network delay and packet loss (Badr et al .2017). The work presented in (Mushtaq et al. 2016) is on the type of data networks, audio codec for transmission and handover during the call. They also tested the application aspects such as the software version, configuration and device characteristics like manufacturer, model, platform and operating system. Their results showed that the device type as well as network parameters have significant impact on the QoE (Mushtaq et al. 2016, De Pessemier et al. 2015). Short call durations has lower ratings compared to long call durations. The influence of the call duration shows the interaction and usage pattern of the end users of VoIP (De Pessemier et al. 2015).

The playout buffer is considered for speech quality and maintains a balance with conservational interactivity. The playout dimension algorithm was used to investigate the objective QoE of MSN messenger, Google talk and Skype; results show that playout buffer is not used properly by these applications. If these applications use playout buffer dimension algorithm then they will provide better QoS and also high QoE provisioning (Wu et al. 2009). QoE/QoS-aware downlink scheduling algorithm was proposed to avoiding delay of network traffic for sensitive VoIP data. The proposed Quality-aware DRX (Q-DRX) Scheme reduced packet losses, increased QoE of user and saved power of user's device. The scheme was compared with previous DRX schemes and the simulation showed that this scheme provides better QoS for improving QoE in terms of power saving (Mushtaq et al. 2015). 
Portugal Telecom implemented ArQoS® for collecting QoS information of network traffic and VoIP calls to assess QoE of VoIP services (Neves et al. 2011). The operating system of the ArQoS $®$ is based on two different modes, non-intrusive mode (passive system) and intrusive mode (active system) (Cardeal et al. 2011). Figure 3 provides details of ArQoS® functions that assessed QoE with the probes. The voice data (RTP packets) captured and processed by probes and prediction of lost and delayed packet are processed by jitter buffer emulator. The distribution and packet loss is measured in Gilbert Model and other networks metrics are also an input to ArQoS ${ }^{\circledR}$ to estimate the QoE of speech quality assessment model.

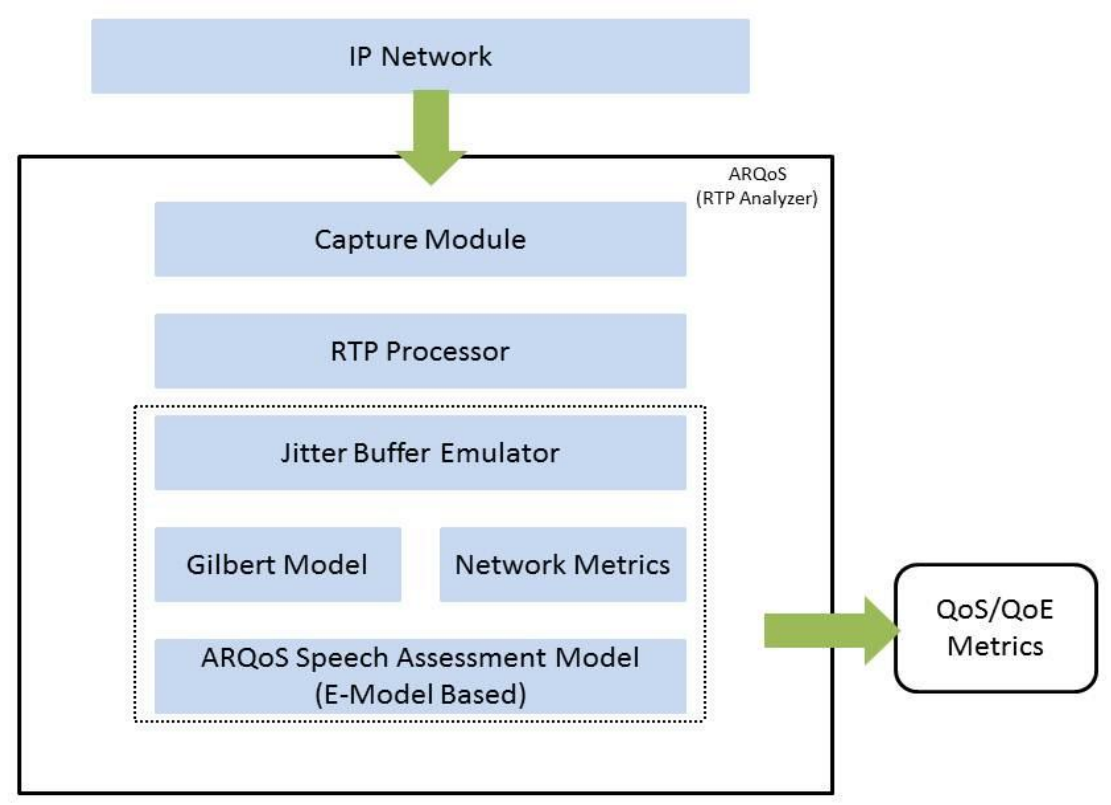

Figure 3. ArQoS® VOIP QoE Monitor (Cardeal et al. 2011)

\subsection{Challenges and Limitations}

Previous research on the VoIP software such as MSN messenger, Gtalk, and Skype increases buffer to provide quality of service but this scheme increases the time duration of the call and user cost per call (Wu et al. 2009). Audio codec, packet delay and loss were used for measuring QoE of the call but no better solution has been provided to avoid such condition during VoIP calls. In addition, service problems also occur in VoIP due to various users in the wireless environment, sometimes wireless networks face numerous challenges such as hand-off delay, signal disturbance and low computing (Regis et al. 2014).

VoIP has features like voicemail to email, remote office, conference calls to multiple people, therefore, future research work will push the industry towards a QoE service provisioning model (Tsolkas et al. 2017), a clear/comprehensive manual on the available parametric models and the critical QoE performance parameters per service type which is currently missing.

\section{Quality of Experience for Web Development}


Internet surfing is a common way for accessing information via the Internet. Users around the world watch videos like news, movies and tutorials, do online shopping and search for information of their particular interest (Scotton et al. 2010, Cui et al. 2012). World Wide Web since late 1990's has been called as World Wide Wait because the users have to wait for a long time to receive a response or load content in the web browser (Shaikh et al. 2012). The web traffic suffers from network issues from host to end user which results in $90 \%$ of users to join other service providers due to low QoS (Pokhrel et al. 2014). The web service providers focus on the measured QoS parameters such as packet loss, delay, reorder, jitter and content loading time although these QoS parameters also affect the QoE (Islam et al. 2014). QoE domain has been added to improve web services for end users and improve their satisfaction level to continue to use services and remain a permanent client (Borchert et al. 2017).

Searching problems degrade user level experience, several QoE assessment research experiments were conducted, one such work provided by (Cui et al. 2012). The researcher proposes K-means clustering to determine the relation between the QoS metrics and subjective QoE. They analyzed four data sessions (clusters) via K-means algorithm to assess the QoE. Another study conducted in different sessions named as signature was based on the QoE metrics and a particular browser plugin was used to measure the QoS data of elements contained by the web page and rendering time. The results show that if round trip delay or packet loss or time for page loading is increased then user dissatisfaction level also increases. This proves that there is a solid correlation between the QoE and loading time; if loading time is increased more than 10 seconds then it is not acceptable for users.

The products purchased via ordering and transferring money via web services from remote locations is growing over the Internet. Providing QoS for loading time of an online shopping site page is also a major problem which degrades the user's QoE. A web based study conducted by Shaikh et al. (2012) shows assessment of QoE of users on different networks delay for online shopping. The experimental setup was created by using Linux based network emulator to disturb the network traffic from server to client as given in Figure 4.

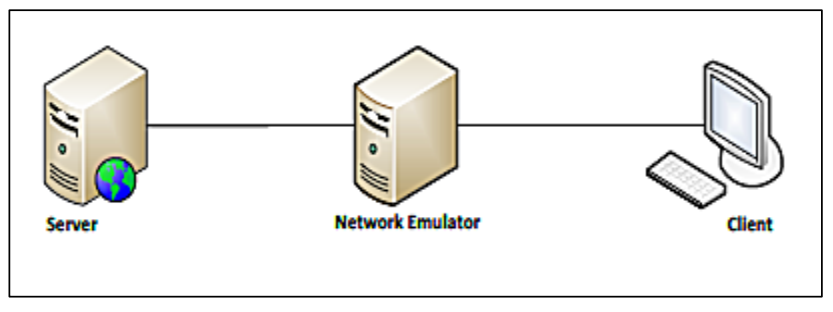

Figure 4. Experiment setup (Shaikh et al. 2012)

The users accessed the web page from the server for online shopping with different delays of $4,10,16$ seconds created in page loading time. The study was conducted in 3 different sessions from individuals via web based shopping. Each session was based on 5 web pages recording the network delay which was different in every session. The subjective study provides results that user assign MOS above 3 on 4 s delay and below 2.5 for $16 \mathrm{~s}$. Their study found that the long loading time of $16 \mathrm{~s}$ is not acceptable for the user during a session.

Cecchet et al. (2013) built mBenchLab software to measure QoE of web services which were hosted by clouds. mBenchlab use real mobile devices instead of an emulator with their own operating system and 
the network interface for performance evaluation. During the experiments, mBenchlab software was used to measure the QoE of popular websites and numerous mobile phones and networks $(3 \mathrm{G}, 4 \mathrm{G}, \mathrm{WiFi})$. The results show that mBenchlab was able to discover bugs which in new smart phones impacts on both data usage and performance.

The web QoE enhanced by developing strategy of packet transmission scheduling in the wireless environment is presented in (Tajima et al. 2016). In the proposed scheme packet transfer was based on the TCP connection handshake increasing the buffer of wireless nodes to store the number of packets. The scheduling of packets was set to display upper part of the web page on a mobile screen so the user could see the top information of the page. The network traffic was emulated by local server and the extra delay was inserted to packets according to latency in sending packets. The QoE was assessed on the time to draw first part or frame of the page (Tajima et al. 2016).

\subsection{Challenges and Limitations}

Several methods are provided by researchers for web QoE assessment and different parameters of the web have been considered during the experiments (Joshi et al. 2016, Bocchi et al. 2017). The results of previous research experiments show that QoE decreases when packet delay is increased and packet loss causes reload of the page which increases the overall loading time of web page (Cui et al. 2012). Increasing the QoE assessment level of user's packet drop scheme is used instead of loading complete page i.e. only upper part of the page is loaded fast for user preview. This scheme also has limitations, if any user's required information is in the lower part of the page then it will have to wait for a longer time to load the page (Tajima et al. 2016).

The QoE of web services for online shopping and content loading was also analyzed to measure QoS but browser software was not considered in the focus for receiving web services at the client side. The user mostly faces problems of flash and java plugin, caching, low resources in their device and slow network speed but these parameters were not considered during the research (Pujol Gil 2017). In the future instead of HTTP and TCP/IP, new protocols such as Multi-Path Transmission Control Protocol (MPTCP) will be added for achieving QoE for web services (Muraki et al. 2017).

\section{Quality of Experience for Games}

Nowadays online gaming is very popular around the world. Users from different locations can easily play multiplayer games via the Internet (Stavropoulos et al. 2017). The online video games are highly profitable network killer applications and consume high bandwidth from server to players (Chang et al. 2010). The user game experience is based on the game development (e.g. design, best or worse competitor in terms of network performance), game server deployment (e.g. client server links keep balance between deployment cost and customer satisfaction) and game play (e.g. depends how many network connections are available at user side like Wi-Fi and DSL which provide better gaming experience) (Ebner and Holzinger 2007, El-Nasr et al. 2016). A number of researchers have analyzed the effect of QoS of network parameters on gaming experience (Chen et al. 2006, Jarschel et al. 2011, 2013, Salih et al. 2016). One such work has done by Ivan et al by testing subjective QoE of the online game (World of Warcraft) using home gaming network (gaming everywhere server) and traditional online gaming (Sliver et al. 2014). During the experiments, impact of network delay and loss conditions were analyzed and results showed that loss and delay significantly impact on the user's QoE. Switching from 
traditional online gaming server to home gaming service has high impact on the QoE due to degradation changes in network conditions in the gaming cloud. Experienced players were not willing to play games under degradation of network conditions as compared to inexperienced players. The user's positive feedback during the experiments also shows that home game streaming is feasible if acceptable video quality is guaranteed when user is playing the game.

Metzger highlighted the importance of frame-rate of video games when user's experience is conducted during experiments (Metzger et al. 2014). Most research work has been done on a single game instead of two or three games, therefore, not examining user's experience diversity. Previous work has not evaluated certain properties of game, such as frame-rate which has a greater impact on QoE. The simulation results show the significance of such properties on the objective measure by putting the End-to-End (E2E) lag in relation to the frame-rate.

Cloud gaming is very popular around the world (Cai et al. 2016). The users do not need high performance computing for rendering game and software license to avoid piracy but require network speed and high definition (HD) display device to play (SanWariya et al. 2016). Jarschel et al evaluated subjective QoE to analyze the effect of the network delay and loss during playing the cloud gaming on the QoE (Jarschel et al. 2011). They selected three games as Pro Evolution Soccer for the omnipresent perspective (slow-pace gameplay), Final Fantasy XIII for the 3rd person perspective (medium-paced gameplay) and Gran Turismo HD Concept for the 1st person perspective (fast-paced gameplay). The purpose of selecting the three games was to provide the players more options to repeat experiments and provide ratings for the games on the different network delay and loss from Server to Client and Client to Server ongoing traffic. NetEm emulator is used in the middle of the gaming cloud followed by client with QoE measuring web server in the test-bed network as given in Figure 5.

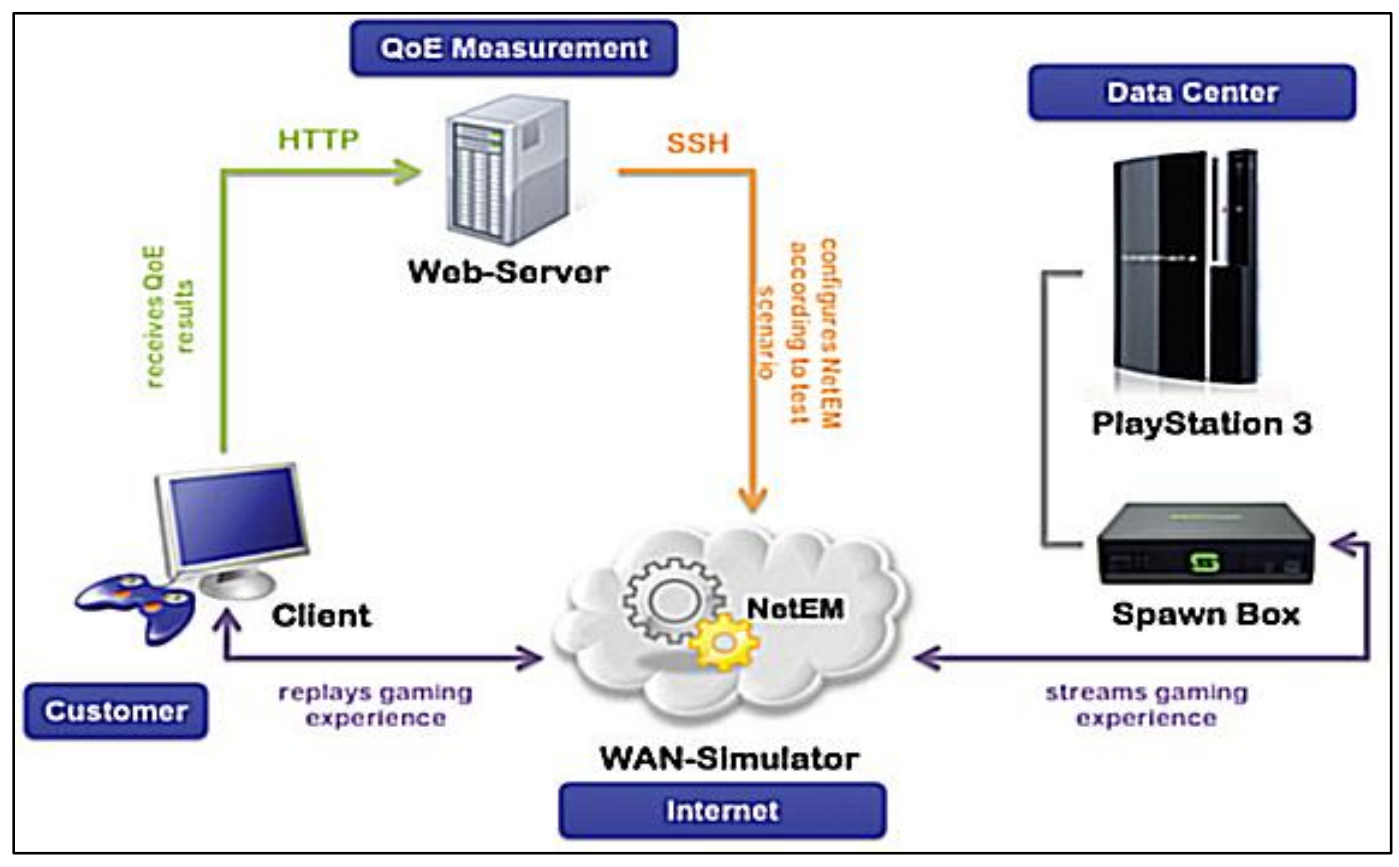

Figure 5. Testbed Setup - Logical View (Cai et al. 2016) 
The results are based on the 79 test runs, 790 users gave ratings for cloud gaming. The MOS values vary from game to game; the slow game gets better user ratings and fast game get poor ratings and medium speed game get fair ratings. The impact of packet loss and delay on cloud gaming is similar to its influence on conventional games. When the network delay (bi-directional) was increased then the user MOS values were decreased because users have to wait to receive a reaction to their input in the game. Players of fast games accept the high loss as compared to high delays because for player's success in the game it is critical to react quickly. During the survey, only $15 \%$ of users were willing to pay the monthly fee for gaming if the quality of service is provided to them.

\subsection{Challenges and Limitations}

QoE is a major factor for analysis of QoS of online gaming because the user satisfaction of game players is important for quality of game delivery from services providers. The research work was done by using the multiple games in different network scenarios where QoE was measured on packet loss and delay. Fast games have low user ratings and slow games have high ratings because fast games require quick response from players but low bi-directional network speed has the influence on fast games. Online multiplayer games require high processing for fast rendering of games and more network bandwidth for sending and receiving data for input actions in games to synchronize play but the slow rendering of graphics at client side decrease the overall QoE of online gaming (George et al. 2017). Cloud computing provides the facility of fast graphics rendering for gaming, multiplayer and only the user require display and control to play but the slow network is a problem for cloud gaming (Wu et al. 2017).

In the future, the addition of speculation-based technology will further improve the delay performance based on the user inputs and past gaming events (Wu et al 2017). The frame rate of games will decrease for better gaming from clouds and also will be adjustable according to network conditions.

\section{Quality of Experience in Cloud Computing}

Cloud computing is an emerging technology that provides computing resources like CPU resources, operating system environment and application development, which are broadly distributed over the Internet (Hashem et al. 2017, Chunlin and Layuan 2014). The cloud computing model of IT services were designed to provide QoS to consumers. SLA is signed between the service provider and consumer for QoS terms and conditions (Al-Shammari, S. and Al-Yasiri 2014, Alhamad et al. 2010, Manuel 2015). The QoS commonly refers to technical parameters that determine the quality of processing, storage and network used for transferring data from cloud to client but does not take the customer into consideration (Abdelmaboud et al. 2015, Singh and Chana 2015). The QoE in contrast, defines the performance of cloud processing, storage and network from the perspective of the customer (Amrehn et al. 2013, Zhu et al. 2011). The QoE domain has also merged in cloud computing to manage and provide services according to customer's demand (Kafetzakis et al. 2012).

There have been many QoE based frameworks proposed for cloud computing for assessment of subjective and objective QoE for cloud services (Wang et al. 2013, Taleb and Ksentini 2013, Costa et al. 2013, Alhamazani et al. 2014). One such is Mobile Cloud Gaming (CMG) framework which was proposed for multi user gaming environment for the mobile user via cloud server instead of client server architecture (Wang and Dey 2012.). The purpose of the framework is to provide an idea to shift mobile user load to cloud server due to the inherent hardware constraint of mobile devices (memory and graphics processing). 
The framework is based on the objective and subjective QoE measures. The objective factors that impact on the QoE are four: cloud server, source video, wireless network and client. The authors proposed network based model for subjective QoE validation of framework and Game Mean Opinion Score (GMOS) for measurement of end user's QoE.

The CloudFog lightweight system was proposed by Yuhua, the Fog is based on the concept of supernodes that are closed to end users and connected to clouds (Lin and Shen 2015). In CloudFog system, cloud conducted the intensive computation of new game state of the virtual world and sends the update to supernodes, the overall scenario is given in Figure 6.

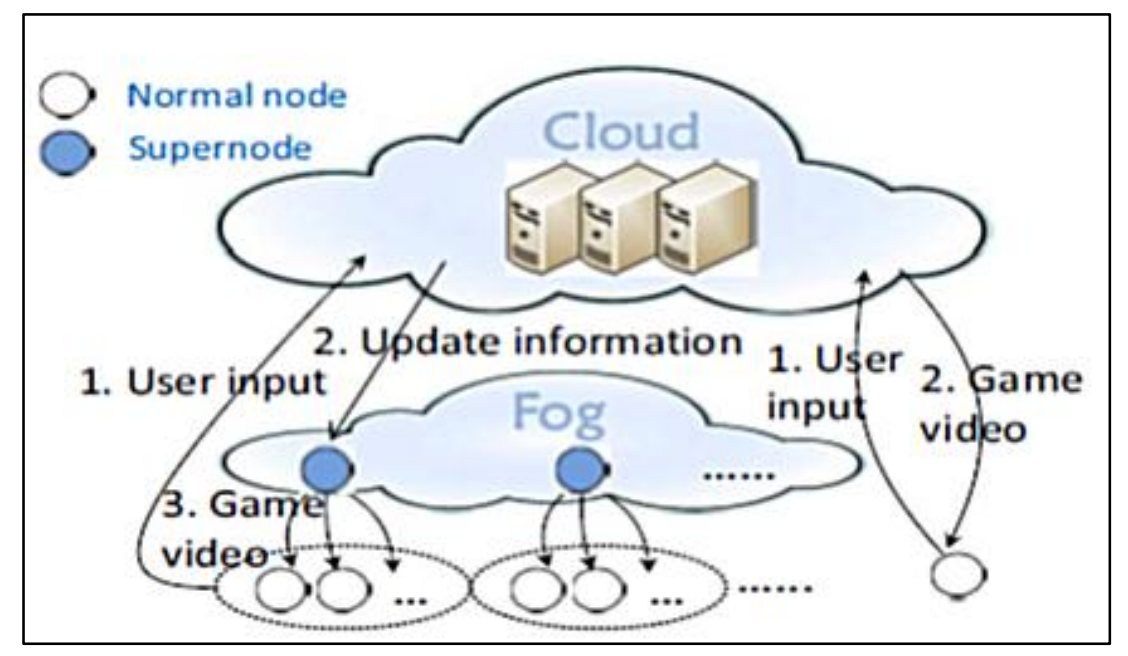

Figure 6. Fog-assisted cloud gaming infrastructure (Lin and Shen 2015)

Supernodes are in the middle of the cloud, the client receives rendered data from the cloud and forwards it to different players. The user does not have high speed network connection to access the cloud which receives an update of the virtual world and renders videos of the game. As a result, cloud does not transfer the entire game video to the long distance users. This technique supports high QoE on low-speed networks, short response time, increase user coverage and reduce bandwidth cost. In addition to the CloudFog techniques in literature that support QoE are Gaming anywhere (Huang et al. 2015) and EdgeCloud (Choy et al. 2012) models. The EdgeCloud model adds a number of servers to perform all the tasks of cloud like rendering, storing and game status, while in CloudFog all computation was done on the cloud and supernodes only receive updates from the cloud and forward to the clients. During the experiment, 2 common datacenters were used, CloudFog used 750 distributed nodes and 300 capacities of supernodes, whereas, EdgeCloud used eight random servers. The EdgeCloud provides the response with less latency as compared to the cloud because of distributed servers as users are connected to the server within the short distance. In the CloudFog users are physically close to supernodes, so the user receives videos from close supernodes instead of far-away servers.

The QoE of Cloud based video streaming for mobile was assessed by Nouha et al. They considered the influence of factors such as media, network, device and content (Samet et al. 2016). Sixty second videos were set on the cloud video server for mobile users. The server will collect influence factors like media source, user terminal, network condition and MOS ratings. Ratings were collected by using different 
network conditions and devices for proper evaluation of user perception. The subjective QoE and objective QoS were used to put in membership functions and inference of fuzzy logic systems.

\subsection{Challenges and Limitations}

The merging of QoE has changed the management strategies of cloud service providers' focus more on QoE instead of QoS technical parameters (Casas and Schatz 2014). The QoE domain is added into cloud computing models to provide QoS to end users but still challenges remain to get QoE of the user on every parameter of service delivery (Hobfeld et al. 2012). The developed QoE models are limited in monitoring all aspects of users and automatically managing services according to current user needs and service status. The proposed CMG framework has limited functionality of cloud environment monitoring and is unable for network and client device with no reporting policy on the services degradation (Wang and Dey 2012). The CloudFog model use supernodes to provide QoS with good QoE for short distance. If the distance is increased between the user and the cloud then degradation in service quality results and also QoE level is decreased (Huang et al. 2015). EdgeCloud uses servers in the nearby location to clients to provide QoS with less delay due to short distance communication but the long distance of users to cloud is still a major problem (Laghari et al. 2016).

In the future, minimization of packet delay and loss will increase the user satisfaction for cloud services and also cloud service provider will give compensation to the user if the QoS of the services are low as per the SLA. The compression of video and image cloud also decreases the QoE of users so the development of new storage algorithms which compresses the size of the file for storage without affecting the quality of the file is better for user satisfaction and also for cloud management (Lin 2016, Castiglione et al. 2015). Integration of social media clouds for video hosting is required for long time duration of HD video hosting. Video sharing and downloading will also increase the QoE of users for quality video access.

\section{Open Research Issues}

There has been lots of work done by researchers on QoE around the world, however, there is still a long way to capture positive and accurate QoE for business management. We summarize the following key areas of research in QoE to advance the work in this area.

QoE essentials: QoE has been described by numerous researchers, standards and procedures for assessment are also given by institutes such as ITU and QUALINET but still, objective QoE assessment methods are not clear. So there is further need to explore qualitative research methods, physiological assessment methods and applications, sensory evaluation, behavioral assessment, statistical methods, and experimental validity (Engelke et al. 2017, Egan et al. 2016).

Image and video QoE analysis: Rapid growth of social media networks and competition of earning revenues between service providers encourage to provide QoS to end users and consider their experience for future development. Image and video sharing is a common feature of social media networks but high quality images and videos require more storage and management so quality, compression, contents analysis of image, video and audios are further consideration for future work (Tao et al. 2015, Paudyal et al. 2017, Laghari et al. 2017). 
Social media contents: Social media networks are the open platform for users around the world to share their ideas in the shape of image, videos, text and audios but users from different communities and geographical areas do not want to see everything. So QoE to content and filter for social media, QoE in communities and social TV are the main factors for QoE assessment and the future research directions (Taleb et al. 2016).

Cloud, network and service management: Cloud and network management is important for service providers to provide QoS to end users, so they can evaluate their services via subjective feedback of users sometimes that contains inaccurate and negative responses (Laghari et al. 2017). Therefore, for proper management accurate and positive QoE is required, leading to further work in QoE monitoring and management. This includes the network environment e.g. fixed or wireless, network conditions such as available bandwidth, packet loss, terminal capabilities, CPU power, resolutions, codec and SLA with network or service operator, specific to service or application analysis of subjective and objective QoE for accuracy and service provision according to SLA (Moreno-Vozmediano et al. 2017). The cloud and network based framework development for controlling QoE in the runtime environment and QoE optimization is also essential (Simoens et al. 2017, Laghari et al. 2017).

QoE domains: The QoE domain is merged in all six areas of applications to improve services according to user needs. The technical parameters are different in every field such as multimedia services contains bitrate of video, frame rate, video codecs etc. VoIP uses audio codecs and cloud computing uses different technical parameters for service delivery such as packet delay, packet loss and user distance from cloud, storage and data retrieval. The CPU uses resource utilization according to the defined SLA. In games, video delivery is either in HD quality or less, user input response for fast and slow games varies. In web technologies time of loading contents in the web-page with image and video quality also varies. In Table 2 we summarize the QoE requirements in different application areas presented in this paper highlighting the QoE requirements and future considerations. This has policy implications in the different application areas presented in this paper. For example, if QoE is benchmarked then vendors can charge for high QoE. However, if the user does not receive the QoE then they can demand a refund on their services and ask for compensation. Therefore, the increase in multimedia traffic over a number of application areas can result in new policies been formulated if QoE is benchmarked. 
Table 2: QoE Requirements for Different Areas.

\begin{tabular}{|c|c|c|}
\hline Applications & QoE Parameters & $\begin{array}{c}\text { Future } \\
\text { considerations }\end{array}$ \\
\hline $\begin{array}{l}\text { Multimedia } \\
\text { services }\end{array}$ & $\begin{array}{l}\text { AQoS (e.g. codec } \\
\text {,frame rate) } \\
\text { NQoS (e.g. } \\
\text { bitrate) }\end{array}$ & $\begin{array}{l}\text { monitoring of } \\
\text { client device, } \\
\text { design of } \\
\text { algorithms for } \\
\text { analysis of } \\
\text { accurate QoE and } \\
\text { network policy } \\
\text { change }\end{array}$ \\
\hline $\begin{array}{l}\text { Network } \\
\text { services }\end{array}$ & $\begin{array}{l}\text { NQoS (Packet } \\
\text { loss \& reorder) }\end{array}$ & $\begin{array}{l}\text { SLAs, automatic } \\
\text { network } \\
\text { monitoring, } \\
\text { dynamic policy }\end{array}$ \\
\hline VoIP & $\begin{array}{c}\text { NQoS (delay, etc) } \\
\& \text { AQoS (audio } \\
\text { codec, etc) }\end{array}$ & $\begin{array}{l}\text { QoE performance } \\
\text { parameters per } \\
\text { service type }\end{array}$ \\
\hline $\begin{array}{c}\text { Web } \\
\text { development }\end{array}$ & $\begin{array}{l}\text { NQoS (loading } \\
\text { time ) }\end{array}$ & $\begin{array}{l}\text { New protocols } \\
\text { such as Multi- } \\
\text { Path Transmission } \\
\text { Control Protocol } \\
\text { (MPTCP) }\end{array}$ \\
\hline Games & $\begin{array}{l}\text { NQoS, AQoS, } \\
\text { PSNR \& VGA }\end{array}$ & $\begin{array}{l}\text { speculation-based } \\
\text { technology }\end{array}$ \\
\hline Cloud & $\begin{array}{c}\text { NQoS \& AQoS } \\
\text { (data retrieval) }\end{array}$ & $\begin{array}{c}\text { SLA, sentiment } \\
\text { analysis }\end{array}$ \\
\hline
\end{tabular}

\section{Conclusion}

In this paper, we presented analysis and start-of-the-art of merging in QoE in different applications to improve customer satisfaction and provide QoS according to their demands. Table 1 provide scale for subjective QoE assessment as given by ITU and Figure 4 represent common network test bed used in several experiments for assessment of QoE on different network parameters for voice and video services. We give key concepts and background of QoE, We also describe its types such as subjective and objective and contextualize use of QoE. We described latest developments in QoE in multimedia services, network management, VoIP, web development, games and cloud computing and a comprehensive literature review. Finally, we have discussed the open issues and future directions for research in the field of QoE.

Acknowledgments: The work is supported by the National Key R\&D Program of China under grant No. 2017YFB0801801, the National Science Foundation of China (NSFC) under grant No. 61472108 and 61672186.

Author Contributions: Author Contributions: The first author has conducted the research and written the paper and the third author has set the template and format of the paper. Rest of the authors have reviewed the paper to set the context and contributed as experts of fields.

Conflicts of Interest: Declare conflicts of interest or state "The authors declare no conflict of interest." 


\section{References}

Dong, M., Kimata, T., Sugiura, K. and Zettsu, K., (2014). Quality-of-experience (QoE) in emerging mobile social networks. IEICE TRANSACTIONS on Information and Systems, 97(10), pp.2606-2612.

Le Callet, P., Möller, S. and Perkis, A., (2013). Qualinet White Paper on Definitions of Quality of Experience (2012). European Network on Quality of Experience in Multimedia Systems and Services (COST Action IC 1003). Version 1.2. Mar-2013.

Laghari, A. A., Sadhayo, I. H., \& Channa, M. I. (2015). Enhanced autonomic networking management architecture (Enama). Engineering, Science \& Technology, 14(1), 9-13.

ITU-T IPTV, F.G., (2007). Definition of quality of experience (QoE).

Laghari, K.U.R. and Connelly, K., (2012). Toward total quality of experience: A QoE model in a communication ecosystem. IEEE Communications Magazine, 50(4).

Nourikhah, H. and Akbari, M.K., (2016). Impact of service quality on user satisfaction: Modeling and estimating distribution of quality of experience using Bayesian data analysis. Electronic Commerce Research and Applications, 17, pp.112-122.

Laghari, A.A., He, H. and Channa, M.I., (2018). Measuring Effect of Packet Reordering on Quality of Experience (QoE) in Video Streaming. 3D Research, 9(3), p.30.

Soldani, D., Li, M. and Cuny, R. eds., (2007). QoS and QoE management in UMTS cellular systems. John Wiley \& Sons..

Khan, A., Sun, L., Ifeachor, E., Fajardo, J.O., Liberal, F. and Koumaras, H., (2010). Video quality prediction models based on video content dynamics for H. 264 video over UMTS networks. International Journal of Digital Multimedia Broadcasting, 2010.

Laghari, A.A., He, H., Shafiq, M. and Khan, A., (2018). Assessment of quality of experience (QoE) of image compression in social cloud computing. Multiagent and Grid Systems, 14(2), pp.125-143.

Varela, M., Skorin-Kapov, L. and Ebrahimi, T., (2014). Quality of service versus quality of experience. In Quality of experience(pp. 85-96). Springer, Cham.

BT, R.I.R., (2002). Methodology for the subjective assessment of the quality of television pictures.

Chen, Y., Wu, K. and Zhang, Q., (2015). From QoS to QoE: A tutorial on video quality assessment. IEEE Communications Surveys \& Tutorials, 17(2), pp.1126-1165.

Laghari, A.A., He, H., Khan, A., Kumar, N. and Kharel, R., (2018). Quality of Experience Framework for Cloud Computing (QoC). IEEE Access.

Zhao, T., Liu, Q. and Chen, C.W., (2017). QoE in video transmission: A user experience-driven strategy. IEEE Communications Surveys \& Tutorials, 19(1), pp.285-302.

Ljubojević, M., Vasković, V. and Starčević, D., (2013). The analysis of the users' response to the linear internet video advertising by using QoE methods. Journal of Universal Computer Science, 19(12), pp.1736-1760. 
Venkataraman, M. and Chatterjee, M., (2011). Inferring video QoE in real time. IEEE network, 25(1).

Kawano, T., Yamagishi, K., Watanabe, K. and Okamoto, J., (2010), December. No reference video-qualityassessment model for video streaming services. In Packet Video Workshop (PV), 2010 18th International (pp. 158164).

Laghari, A.A., He, H., Zardari, S. and Shafiq, M., (2017). Systematic Analysis of Quality of Experience (QoE) Frameworks for Multimedia Services. IJCSNS, 17(5), p.121.

Laghari, A. A., Pham, T.T., Nguyen, H. and Crespi, N., (2012). QoM: A new quality of experience framework for multimedia services. In Computers and Communications (ISCC), 2012 IEEE Symposium on (pp. 000851-000856).

Laghari, A.A., Channa, M.I., Laghari, K.R., Aman, M. and Memon, M., (2013). EQOM: enhanced quality of experience (QoE) framework for multimedia services. UACEE International Journal of Computer Science and its Applications, 3(1), pp.85-89.

Laghari, A.A., He, H., Ibrahim, M. and Shaikh, S., (2017). Automatic Network Policy Change on the Basis of Quality of Experience (QoE). Procedia Computer Science, 107, pp.657-659.

http://www.peerapp.com/quality-of-experience/ [accessed 10/19/16]

Oche, M., Noor, R.M. and Chembe, C., (2017). Multivariate statistical approach for estimating QoE of real-time multimedia applications in vehicular ITS network. Computer Communications, 104, pp.88-107.

Laghari, A.A., He, H., Khan, A. and Karim, S., (2018). Impact of Video File Format on Quality of Experience (QoE) of Multimedia Content. 3D Research, 9(3), p.39.

Varga, N., Bokor, L. and Piri, E., (2016). A network-assisted flow mobility architecture for optimized mobile medical multimedia transmission. Annals of Telecommunications, 71(9-10), pp.489-502.

Shaikh, J., Fiedler, M. and Collange, D., (2010). Quality of experience from user and network perspectives. annals of telecommunications-annales des telecommunications, 65(1-2), pp.47-57.

Laghari, U. K., Crespi, N., Molina, B. and Palau, C.E., (2011). QoE aware service delivery in distributed environment. In Advanced information networking and applications (WAINA), 2011 IEEE Workshops of International Conference on (pp. 837-842).

Reichl, P., (2010). From charging for quality of service to charging for quality of experience. annals of telecommunications-annales des télécommunications, 65(3-4), pp.189-199.

Khan, A., Sun, L., Jammeh, E. and Ifeachor, E., (2009). Content classification-based and QoE-driven video send bitrate adaptation scheme. In Proceedings of the 5th International ICST Mobile Multimedia Communications Conference (p. 16). ICST (Institute for Computer Sciences, Social-Informatics and Telecommunications Engineering).

Baraković, S. and Skorin-Kapov, L., (2013). Survey and challenges of QoE management issues in wireless networks. Journal of Computer Networks and Communications, 2013.

Varela, M., Zwickl, P., Reichl, P., Xie, M. and Schulzrinne, H., (2015), June. From service level agreements (SLA) to experience level agreements (ELA): The challenges of selling QoE to the user. In Communication Workshop (ICCW), 2015 IEEE International Conference on (pp. 1741-1746) 
Menkovski, V. and Liotta, A., (2012). QoE for mobile streaming. Mobile multimedia: user and technology perspectives.

https://www.spirent.com/Newsroom/Press_Releases/Releases/2016/February/02-24-16_Spirent-assures-QoE-andbusiness-critical-SLAs-for-Wifi-ethernet-and-lte [accessed 10/19/16]

Laghari, A.A., Laghari, K.U.R., Channa, M.I. and Falk, T.H., (2012). QON: Quality of experience (QoE) framework for network services. In Proceedings of the 4th International Conference on Software Technology and Engineering (ICSTE'12)..

Mongi, A.F., (2015). A Conceptual Framework for QoE Measurement and Management in Networked Systems. International Journal of Computer Applications, 112(8).

Phan-Xuan, T. and Kamioka, E., (2016). Efficiency of QoE-driven network management in adaptive streaming over HTTP. In Communications (APCC), 2016 22nd Asia-Pacific Conference on (pp. 517-522). IEEE..

Phan-Xuan, T. and Kamioka, E., (2016). A machine learning based network monitoring and management system. In The 10th South East Asian Technical University Symposium, Tokyo, Japan.

Hossain, E. and Hasan, M., (2015). 5G cellular: key enabling technologies and research challenges. IEEE Instrumentation \& Measurement Magazine, 18(3), pp.11-21.

Lloret, J., Canovas, A., Tomas, J. and Atenas, M.,( 2012). A network management algorithm and protocol for improving QoE in mobile IPTV. Computer Communications, 35(15), pp.1855-1870.

Nihei, K., Satoda, K. and Yoshida, H., (2016). A QoE indicator and a transmission control method for VoIP on mobile networks considering delay spikes. In Communications (ICC), 2016 IEEE International Conference on (pp. 1-6).

Jones, W.S., Cotton, T. and Holland, R.V., Motorola Solutions Inc, (2000). Voice over internet protocol telephone system and method. U.S. Patent 6,141,341.

Papadopouli, M., Charonyktakis, P., Plakia, M. and Tsamardinos, I., (2015). On user-centric modular qoe prediction for voip based on machine-learning algorithms. IEEE Transactions on mobile computing, (1), pp.1-1..

Badr, A., Khisti, A., Tan, W.T., Zhu, X. and Apostolopoulos, J., (2017). FEC for VoIP using dual-delay streaming codes. In INFOCOM 2017-IEEE Conference on Computer Communications, IEEE (pp. 1-9). IEEE..

Mushtaq, M.S., Fowler, S., Augustin, B. and Mellouk, A., (2016). QoE in 5G cloud networks using multimedia services. In Wireless Communications and Networking Conference (WCNC), 2016 IEEE (pp. 1-6).

De Pessemier, T., Stevens, I., De Marez, L., Martens, L. and Joseph, W., (2015). Analysis of the quality of experience of a commercial voice-over-IP service. Multimedia Tools and Applications, 74(15), pp.5873-5895.

Wu, C.C., Chen, K.T., Huang, C.Y. and Lei, C.L., (2009). An empirical evaluation of VoIP playout buffer dimensioning in Skype, Google talk, and MSN Messenger. In Proceedings of the 18th international workshop on Network and operating systems support for digital audio and video (pp. 97-102). ACM.

Mushtaq, M.S., Fowler, S., Mellouk, A. and Augustin, B., (2015). QoE/QoS-aware LTE downlink scheduler for VoIP with power saving. Journal of Network and Computer Applications, 51, pp.29-46.

Neves, F., Cardeal, S., Soares, S., Assuncao, P. and Tavares, F., (2011). Quality model for monitoring QoE in VoIP services. In EUROCON-International Conference on Computer as a Tool (EUROCON), 2011 IEEE (pp. 1-4). 
Cardeal, S., Neves, F., Soares, S., Tavares, F. and Assunção, P., (2011). Arqos®: system to monitor qos/qoe in voip. In EUROCON-International Conference on Computer as a Tool (EUROCON), 2011 IEEE (pp. 1-2).

Regis Jr, J., (2014). Securing VoIP: Keeping Your VoIP Network Safe. Elsevier.

Tsolkas, D., Liotou, E., Passas, N. and Merakos, L., (2017). A survey on parametric QoE estimation for popular services. Journal of Network and Computer Applications, 77, pp.1-17.

Scotton, J., Moebs, S., McManis, J. and Cristea, A.I., (2010). Merging Strategies for Authoring QoE-based Adaptive Hypermedia. J. UCS, 16(19), pp.2756-2779.

Cui, H. and Biersack, E., (2012). On the relationship between QoS and QoE for web sessions. EURECOM, Sophia Antipolis, France, Tech. Rep. RR-12-263.

Shaikh, J., (2012). Non-Intrusive Network-Based Estimation of Web Quality of Experience Indicators (Doctoral dissertation, Blekinge Institute of Technology).

Pokhrel, J., Lalanne, F., Cavalli, A. and Mallouli, W., (2014). QoE estimation for web service selection using a Fuzzy-Rough hybrid expert system. In Advanced Information Networking and Applications (AINA), 2014 IEEE 28th International Conference on (pp. 629-634).

Islam, N., Elepe, V.J.D., Shaikh, J. and Fiedler, M., (2014). In small chunks or all at once? User preferences of network delays in web browsing sessions. In IEEE International Conference on Communications Workshops (ICC), Sydney. IEEE..

Borchert, K., Hirth, M., Zinner, T. and Göritz, A., (2017), August. Designing a Survey Tool for Monitoring Enterprise QoE. In Proceedings of the Workshop on QoE-based Analysis and Management of Data Communication Networks (pp. 37-42). ACM..

Cecchet, E., Sims, R., He, X. and Shenoy, P., (2013). mBenchLab: Measuring QoE of Web applications using mobile devices. In Quality of Service (IWQoS), 2013 IEEE/ACM 21 st International Symposium on (pp. 1-10).

Tajima, T. and Okabe, Y., (2016). Optimizing Packet Transmission Scheduling for Enhanced Web QoE in Wireless LAN. In Computer Software and Applications Conference (COMPSAC), 2016 IEEE 40th Annual (Vol. 2, pp. 312318).

Joshi, S.S. and Ramanaiah, O.B.V., (2016). An integrated QoE and QoS based approach for web service selection. In ICT in Business Industry \& Government (ICTBIG), International Conference on (pp. 1-7).

Bocchi, E., De Cicco, L., Mellia, M. and Rossi, D., (2017). The web, the users, and the mos: Influence of http/2 on user experience. In International Conference on Passive and Active Network Measurement (pp. 47-59).

Pujol Gil, E., (2017). Web content delivery, monetization, and search.

Muraki, Y., Ito, Y. and Tashiro, K., (2017). Study on MPTCP Enhancement by IP Routing for WebQoE Improvement. In Platform Technology and Service (PlatCon), 2017 International Conference on (pp. 1-4).

Stavropoulos, V., Kuss, D.J., Griffiths, M.D., Wilson, P. and Motti-Stefanidi, F., (2017). MMORPG gaming and hostility predict Internet addiction symptoms in adolescents: An empirical multilevel longitudinal study. Addictive behaviors, 64, pp.294-300. 
Chang, Y.C., Chen, K.T., Wu, C.C., Ho, C.J. and Lei, C.L., (2010). Online game QoE evaluation using paired comparisons. In Communications Quality and Reliability (CQR), 2010 IEEE International Workshop Technical Committee on (pp. 1-6).

Ebner, M. and Holzinger, A., (2007). Successful implementation of user-centered game based learning in higher education: An example from civil engineering. Computers \& education, 49(3), pp.873-890.

El-Nasr, M.S., Drachen, A. and Canossa, A., (2016). Game analytics. Springer London Limited.

Chen, K.T., Huang, P. and Lei, C.L., (2006). How sensitive are online gamers to network quality?. Communications of the ACM, 49(11), pp.34-38.

Jarschel, M., Schlosser, D., Scheuring, S. and Hoßfeld, T., (2011). An evaluation of QoE in cloud gaming based on subjective tests. In Innovative mobile and internet services in ubiquitous computing (imis), 2011 fifth international conference on (pp. 330-335).

Jarschel, M., Schlosser, D., Scheuring, S. and Hoßfeld, T.,(2013). Gaming in the clouds: QoE and the users' perspective. Mathematical and Computer Modelling, 57(11-12), pp.2883-2894.

Salih, Y.K., See, O.H. and Ibrahim, R.W., (2016). An intelligent selection method based on game theory in heterogeneous wireless networks. Transactions on emerging telecommunications technologies, 27(12), pp.16411652

Slivar, I., Suznjevic, M., Skorin-Kapov, L. and Matijasevic, M., (2014). Empirical QoE study of in-home streaming of online games. In Network and Systems Support for Games (NetGames), 2014 13th Annual Workshop on (pp. 1-6).

Metzger, F., Rafetseder, A., Schwartz, C. and Hoßfeld, T., (2016). Games and frames: A strange tale of qoe studies. In Proceedings of the International Conference on Quality of Multimedia Experience, Lisbon, Portugal.

Cai, W., Shea, R., Huang, C., Chen, K., Liu, J., Leung, V.C. and Hsu, C., (2016). The future of cloud gaming. Proceedings of the IEEE, 104(4), pp.687-691.

SanWariya, A., Nair, R. and Shiwani, S., (2016). Analyzing processing overhead of Type-0 hypervisor for cloud gaming. In Advances in Computing, Communication, \& Automation (ICACCA)(Spring), International Conference on(pp. 1-5).

George, M.M. and George, J.M., Amazon Technologies Inc, (2017). Determining real-world effects from games. U.S. Patent 9,597,584.

Wu, J., Yuen, C., Cheung, N.M., Chen, J. and Chen, C.W., (2017). Streaming Mobile Cloud Gaming Video Over TCP With Adaptive Source-FEC Coding. IEEE Transactions on Circuits and Systems for Video Technology, 27(1), pp.32-48.

Hashem, I.A.T., Yaqoob, I., Anuar, N.B., Mokhtar, S., Gani, A. and Khan, S.U., (2015). The rise of "big data" on cloud computing: Review and open research issues. Information Systems, 47, pp.98-115.

Chunlin, L. and Layuan, L., (2014). Multi-layer resource management in cloud computing. Journal of network and systems management, 22(1), pp.100-120..

Al-Shammari, S. and Al-Yasiri, A., (2014). Defining a metric for measuring QoE of SaaS cloud computing. Proceedings of PGNET, pp.251-256. 
Alhamad, M., Dillon, T. and Chang, E., (2010). Sla-based trust model for cloud computing. In Network-Based Information Systems (NBiS), 2010 13th International Conference on (pp. 321-324).

Manuel, P., (2015). A trust model of cloud computing based on Quality of Service. Annals of Operations Research, 233(1), pp.281-292.

Abdelmaboud, A., Jawawi, D.N., Ghani, I., Elsafi, A. and Kitchenham, B., (2015). Quality of service approaches in cloud computing: A systematic mapping study. Journal of Systems and Software, 101, pp.159-179..

Singh, S. and Chana, I., (2015). Q-aware: Quality of service based cloud resource provisioning. Computers \& Electrical Engineering, 47, pp.138-160.

Amrehn, P., Vandenbroucke, K., Hoßfeld, T., Moor, K.D., Hirth, M., Schatz, R. and Casas, P., (2013). Need for speed? on quality of experience for cloud-based file storage services. Proceedings of the PQS, pp.184-190.

Zhu, W., Luo, C., Wang, J. and Li, S., (2011). Multimedia cloud computing. IEEE Signal Processing Magazine, 28(3), pp.59-69.

Kafetzakis, E., Koumaras, H., Kourtis, M.A. and Koumaras, V., (2012). QoE4CLOUD: A QoE-driven multidimensional framework for cloud environments. In Telecommunications and Multimedia (TEMU), 2012 International Conference on(pp. 77-82).

Wang, X., Kwon, T., Choi, Y., Wang, H. and Liu, J., (2013). Cloud-assisted adaptive video streaming and socialaware video prefetching for mobile users. IEEE wireless communications, 20(3), pp.72-79.

Taleb, T. and Ksentini, A., (2013). Follow me cloud: interworking federated clouds and distributed mobile networks. IEEE Network, 27(5), pp.12-19.

Costa, P.M., Pitt, J., Falcão e Cunha, J. and Galvão, T., ( 2012). Cloud2Bubble: enhancing quality of experience in mobile cloud computing settings. In Proceedings of the third ACM workshop on Mobile cloud computing and services (pp. 45-52). ACM.

Alhamazani, K., Ranjan, R., Jayaraman, P.P., Mitra, K., Wang, M., Huang, Z.G., Wang, L. and Rabhi, F., (2014). Real-time qos monitoring for cloud-based big data analytics applications in mobile environments. In Mobile Data Management (MDM), 2014 IEEE 15th International Conference on (Vol. 1, pp. 337-340).

Wang, S. and Dey, S., (2012). Cloud mobile gaming: Modeling and measuring user experience in mobile wireless networks. ACM SIGMOBILE Mobile Computing and Communications Review, 16(1), pp.10-21.

Lin, Y. and Shen, H., (2015). Leveraging fog to extend cloud gaming for thin-client MMOG with high quality of experience. In Distributed Computing Systems (ICDCS), 2015 IEEE 35th International Conference on (pp. 734735). IEEE.

Huang, C.Y., Hsu, C.H. and Chen, K.T., (2015). Gaminganywhere: An open-source cloud gaming platform. ACM SIGMultimedia Records, 7(1), pp.3-5.

Choy, S., Wong, B., Simon, G. and Rosenberg, C., (2012). Edgecloud: A new hybrid platform for on-demand gaming. School Comput. Sci., Univ. Waterloo, Waterloo, ON, USA, Tech. Rep. CS-2012-19.

Samet, N., Letaïfa, A.B., Hamdi, M. and Tabbane, S., (2016). Real-Time User Experience Evaluation for CloudBased Mobile Video. In Advanced Information Networking and Applications Workshops (WAINA), 2016 30th International Conference on (pp. 204-208). 
Casas, P. and Schatz, R., (2014). Quality of experience in cloud services: Survey and measurements. Computer Networks, 68, pp.149-165.

Hobfeld, T., Schatz, R., Varela, M. and Timmerer, C., (2012). Challenges of QoE management for cloud applications. IEEE Communications Magazine, 50(4).

Laghari, A.A., He, H., Shafiq, M. and Khan, A., (2016). Assessing effect of Cloud distance on end user's Quality of Experience (QoE). In Computer and Communications (ICCC), 2016 2nd IEEE International Conference on (pp. 500-505).

Lin, N., (2016). Service-oriented mobile multimedia cooperative storing and delivery scheme based on opportunistic cloud coding and content-centric cloud compression. EURASIP Journal on Wireless Communications and Networking, 2016(1), p.151.

Castiglione, A., Pizzolante, R., De Santis, A., Carpentieri, B., Castiglione, A. and Palmieri, F., (2015). Cloud-based adaptive compression and secure management services for 3D healthcare data. Future Generation Computer Systems, 43, pp.120-134.

Engelke, U., Darcy, D.P., Mulliken, G.H., Bosse, S., Martini, M.G., Arndt, S., Antons, J.N., Chan, K.Y., Ramzan, N. and Brunnström, K., (2017). Psychophysiology-based QoE assessment: A survey. IEEE Journal of Selected Topics in Signal Processing, 11(1), pp.6-21.

Egan, D., Brennan, S., Barrett, J., Qiao, Y., Timmerer, C. and Murray, N., (2016). An evaluation of Heart Rate and ElectroDermal Activity as an objective QoE evaluation method for immersive virtual reality environments. In Quality of Multimedia Experience (QoMEX), 2016 Eighth International Conference on (pp. 1-6).

Tao, X., Dong, L., Li, Y., Zhou, J., Ge, N. and Lu, J.,( 2015). Real-time personalized content catering via viewer sentiment feedback: a QoE perspective. IEEE Network, 29(6), pp.14-19.

Paudyal, P., Battisti, F., Sjöström, M., Olsson, R. and Carli, M., (2017). Towards the perceptual quality evaluation of compressed light field images. IEEE Transactions on Broadcasting, 63(3), pp.507-522..

Laghari, A.A., He, H., Karim, S., Shah, H.A. and Karn, N.K., (2017). Quality of Experience Assessment of Video Quality in Social Clouds. Wireless Communications and Mobile Computing, 2017.

Taleb, T., Ksentini, A., Chen, M. and Jantti, R., (2016). Coping with emerging mobile social media applications through dynamic service function chaining. IEEE Transactions on Wireless Communications, 15(4), pp.2859-2871.

Laghari, A.A., He, H., Shafiq, M. and Khan, A., (2017), May. Impact of storage of mobile on quality of experience (QoE) at user level accessing cloud. In Communication Software and Networks (ICCSN), 2017 IEEE 9th International Conference on pp. 1402-1409.

Moreno-Vozmediano, R., Montero, R.S., Huedo, E. and Llorente, I.M., (2017). Implementation and provisioning of federated networks in hybrid clouds. Journal of Grid Computing, 15(2), pp.141-160.

Simoens, P., Griffin, D., Maini, E., Phan, T.K., Rio, M., Vermoesen, L., Vandeputte, F., Schamel, F. and Burstzynowski, D., (2017). Service-centric networking for distributed heterogeneous clouds. IEEE Communications Magazine, 55(7), pp.208-215.

Laghari, A.A., He, H., Halepoto, I.A., Memon, M.S. and Parveen, S., 2017. Analysis of Quality of Experience Frameworks for Cloud Computing. IJCSNS, 17(12), p.228. 